\title{
Eficiência de controle de cipó-de-veado por glyphosate e glyphosate + 2,4-D em diferentes horários de aplicação ${ }^{1}$
}

\author{
Control efficiency of black bindweed by glyphosate and glyphosate $+2,4-D$ on \\ different application times
}

Cleber Daniel de Goes Maciel²; Cesar Eduardo Lourenço Iuchemin ${ }^{3} ;$ Michel Vicentin de Souza ${ }^{3}$; André Augusto Pazinato da Silva ${ }^{4}$; Ricardo André Kloster Karpinski ${ }^{4}$; Enelise Osco Helvig ${ }^{4}$; Paula Karine Kloster Karpinski ${ }^{5}$; Bruna Teixeira Baixo ${ }^{5}$; João Paulo Matias ${ }^{6}$

Resumo - As aplicações de herbicidas são normalmente realizadas em condições ambientais específicas que podem influenciar a eficácia dos produtos. Este trabalho foi realizado com o objetivo de avaliar a influência de horários de aplicação dos herbicidas glyphosate e glyphosate + 2,4-D no controle da planta daninha cipó-de-veado (Polygonum convolvulus). O experimento foi desenvolvido com vasos em condições de campo. Os tratamentos foram constituídos pelos herbicidas glyphosate $\left(960 \mathrm{~g} \mathrm{ha}^{-1}\right)$ e glyphosate $+2,4-\mathrm{D}\left(960+335 \mathrm{~g} \mathrm{ha}^{-1}\right)$, ambos aplicados em 25 e 26/09/2015, nos horários de 22:00 h, 3:00 h, 7:00 h; 12:00 h e 17:00 h e uma testemunha sem aplicação, para o controle da planta daninha cipó-de-veado. As unidades experimentais foram constituídas por vasos plásticos com 4 plantas em estádio de 3 a 5 folhas, mantidas em condições naturais da aplicação até os 28 DAA (dias após aplicação). As aplicações foram realizadas com um pulverizador costal com quatro pontas de pulverização AVI110.02, pressão de $210 \mathrm{kPa}$, velocidade de deslocamento de $3,6 \mathrm{~km} \mathrm{~h}^{-1}$ e taxa de aplicação de $200 \mathrm{~L} \mathrm{ha}^{-1}$. Os resultados indicaram que glyphosate e glyphosate $+2,4-\mathrm{D}$ controlaram eficientemente a planta daninha a partir dos 14 DAA, independentemente do horário da aplicação. Entretanto, foram constatadas reduções significativa na eficiência de controle e produção de matéria seca da parte aérea da planta daninha no horário das 12:00 h, assim como menor deposição de glyphosate + 2,4-D, em todos os horários das aplicações.

Palavras-chaves: deposição; Polygonum convolvulus; tecnologia de aplicação

Abstract - Herbicides applications are usually held with specific environmental conditions that may influence the efficacy of these products. This research was conducted to evaluate the influence of application times of glyphosate and glyphosate +2.4-D herbicides in Polygonum convolvulus weed control. The experiment was developed with vases arranged in field conditions. The treatments were constituted by glyphosate $\left(960 \mathrm{~g} \mathrm{ha}^{-1}\right)$ and glyphosate $+2.4-\mathrm{D}\left(960+335 \mathrm{~g} \mathrm{ha}^{-1}\right)$

\footnotetext{
${ }^{1}$ Recebido para publicação em 12/10/2016 e aceito em 05/12/2016.

${ }^{2}$ Professor Adjunto do Departamento de Agronomia da Universidade Estadual do Centro Oeste - UNICENTRO. Guarapuava, Paraná, Brasil. E-mail: <cmaciel@ unicentro.br>.

${ }^{3}$ Bolsistas do Programa Institucional de Bolsas de Iniciação Científica para o Ensino Médio (PIBIC-EM) na UNICENTRO. Guarapuava, Paraná, Brasil.

${ }^{4}$ Discentes do Programa de Pós-graduação em Produção Vegetal (PPGA) - UNICENTRO. Guarapuava, Paraná, Brasil.

${ }^{5}$ Acadêmicas do Curso de Agronomia da UNICENTRO. Guarapuava, Paraná, Brasil.

${ }^{6}$ Discente do Programa de Pós-graduação em Ciência Agrárias (PAG) da Universidade Estadual de Maringá - UEM. Umuarama, Paraná, Brasil.
} 
herbicides, both applied in 25 and 9/26/2015, in 10:00 PM hour, 03:00 AM hour, 7:00 AM hour; mid day hour and 5:00 PM hour and a check without application, for controlling P. convolvulus weed. Experimental units were constituted by plastic vases with four plants in three to five leaves stage, kept in natural conditions of application until 28 DAA (days after application). Applications were conducted with a costal spray with four spray nozzles AVI 110.02, $210 \mathrm{kPa}$ pressure, displacement speed of $3.6 \mathrm{~km} \mathrm{~h}^{-1}$ and an application applying rate of $200 \mathrm{~L} \mathrm{ha}^{-1}$. Results indicated that glyphosate and glyphosate + 2.4-D controlled efficiently the weed from 14 DAA, regardless of application hour. However, significant reductions were observed in control efficiency and dry matter production of weed at noon, as well as lower spray deposition of glyphosate $+2.4-\mathrm{D}$, at all times.

Keywords: deposition; Polygonum convolvulus; application technology

\section{Introdução}

A tecnologia de aplicação de produtos fitossanitários ainda é bastante negligenciada por muitos produtores, uma vez que muitas vezes não conseguem produzir uma aplicação com gotas que sejam adequadas para produzir uma boa cobertura do alvo. Somada a essa problemática, o horário de aplicação de agrotóxicos como os herbicidas pode sofrer influência das condições ambientais, afetando o desempenho do produto.

As condições ambientais no momento da aplicação influenciam em diversas etapas da interação herbicida-planta, destacando-se interceptação, absorção e translocação do produto pela planta, podendo ter efeito positivo ou negativo nessas interações (Cieslik et al., 2012; 2013). De forma geral, as condições meteorológicas ideais para as aplicações de fitossanitários são estabelecidas como temperatura abaixo de $30^{\circ} \mathrm{C}$, umidade relativa acima de 55\% e velocidade do vento entre 3 e $12 \mathrm{~km} \mathrm{~h}^{-1}$ (Cunha et al., 2016).

O tempo de vida de uma gota, em sua trajetória entre a ponta de pulverização e o alvo biológico, dependendo do seu tamanho, é até três vezes maior quando a aplicação é realizada a $20^{\circ} \mathrm{C}$ de temperatura e $80 \%$ de umidade relativa do ar, em relação a $30^{\circ} \mathrm{C}$ e $50 \%$, respectivamente (Matthews, 2000). Segundo Antuniassi (2005), ao lidar com as condições do clima, é importante considerar que a ausência de vento também pode ser prejudicial para a deposição de pequenas gotas, devido a correntes de ar quente ascendente. Nesse sentido, o início da manhã e final da tarde são períodos onde a umidade relativa do ar é mais elevada e a temperatura do ar é menor, sendo estes intervalos considerados os mais adequados para as aplicações.

Aplicações matutinas, vespertinas ou noturnas são normalmente realizada com condições ambientais específicas que podem influenciar positiva ou negativamente a eficácia dos herbicidas. Segundo Ramires et al. (1999), a absorção foliar é um fator determinante na ação dos herbicidas aplicados em pósemergência, podendo ser influenciada por vários fatores ambientais, como temperatura, luminosidade, umidade relativa do ar, ventos, presença de orvalho e de chuva, os quais determinarão a maior ou menor permeabilidade da cutícula das plantas e, em consequência, na absorção do produto.

A planta daninha Polygonum convolvulus L., pertence à família Polygonaceae, e constitui uma espécie herbácea anual que se desenvolve nas regiões Sudeste e Sul do Brasil, ocupando com muita frequência áreas olerícolas, lavouras de cereais de inverno ou perenes como pomares de pêssego, onde cresce enrolando-se às plantas sob cultivo (Moreira e Bragança, 2011). É altamente prolífica, com capacidade de uma única planta produzir até 30 mil sementes com capacidade de dormência e viabilidade prolongada no solo, e tem nos cereais de inverno o controle dificultado devido a germinação tardia. Além dos prejuízos por competição, o emaranhamento nos colmos 
dos cereais de inverno favorece o acamamento da cultura, dificultando a colheita mecanizada (Kissmann e Groth, 2000).

No intuito de conciliar a tecnologia de aplicação existente com um horário de maior eficiência, o presente trabalho teve como objetivo estudar a influência de diferentes horários de aplicação dos herbicidas glyphosate e glyphosate + 2,4-D no controle da planta daninha cipó-de-veado ( $P$. convolvulus).

\section{Material e Métodos}

Um experimento foi desenvolvido no campus da Universidade Estadual do CentroOeste, Guarapuava/PR, em área localizada nas coordenadas geográficas S $23^{\circ} 12 ’ 28,8^{\prime \prime}$ de latitude, W 53ำ'14,7" de longitude e a $1020 \mathrm{~m}$ de altitude, utilizando como unidades experimentais vasos plásticos de $3 \mathrm{~kg}$ de solo com 4 plantas de cipó-de-veado $(P$. convolvulus), mantidas diretamente a campo.

Onze tratamentos foram constituídos pelos herbicidas glyphosate $\left(960 \mathrm{~g} \mathrm{ha}^{-1}\right) \mathrm{e}$ glyphosate + 2,4-D $\left(960+335 \mathrm{~g} \mathrm{ha}^{-1}\right)$, ambos aplicados em 25/09/2015, nos horários de 22:00 h, 3:00 h, 7:00 h; 12:00 h e 17:00 h e uma testemunha sem aplicação (Tabela 1), visando a deposição da aplicação e o controle das plantas de cipó-de-veado. O delineamento experimental utilizando foi o inteiramente casualizado, com cinco e dez repetições para as avaliações de controle e deposição da aplicação, respectivamente.

Tabela 1. Herbicidas, horários de aplicação e condições ambientais que constituíram os tratamentos utilizados nas avaliações de deposição da aplicação e o controle da planta daninha cipó-de-veado. Guarapuava (PR), 2015.

\begin{tabular}{|c|c|c|}
\hline Herbicidas (dose) & Horários & Condições Ambientais \\
\hline 1. Glyphosate $\left(960 \mathrm{~g} \mathrm{ha}^{-1}\right)$ & & Temp $=25,2^{\circ} \mathrm{C} ; \mathrm{UR}=51,3 \%$ \\
\hline 2. Glyphosate + 2,4-D $\left(960+335 \mathrm{~g} \mathrm{ha}^{-1}\right)$ & $22: 00 \mathrm{~h}$ & $\mathrm{~V}=2,1 \mathrm{~km} \mathrm{~h}^{-1} ;$ (ausência nuvens) \\
\hline 3. Glyphosate $\left(960 \mathrm{~g} \mathrm{ha}^{-1}\right)$ & & Temp $=19,4^{\circ} \mathrm{C} ; \mathrm{UR}=63,9 \%$ \\
\hline 4. Glyphosate + 2,4-D $\left(960+335 \mathrm{~g} \mathrm{ha}^{-1}\right)$ & $3: 00 \mathrm{~h}$ & $\mathrm{~V}=1,8 \mathrm{~km} \mathrm{~h}^{-1} ;$ (neblina) \\
\hline 5. Glyphosate $\left(960 \mathrm{~g} \mathrm{ha}^{-1}\right)$ & & Temp $=22,0^{\circ} \mathrm{C} ; \mathrm{UR}=65,3 \%$; \\
\hline 6. Glyphosate $+2,4-\mathrm{D}\left(960+335 \mathrm{~g} \mathrm{ha}^{-1}\right)$ & $7: 00 \mathrm{~h}$ & $\mathrm{~V}=1,2 \mathrm{~km} \mathrm{~h}^{-1} ;$ (leve orvalho) \\
\hline 7. Glyphosate $\left(960 \mathrm{~g} \mathrm{ha}^{-1}\right)$ & & $\mathrm{Temp}=26,3^{\circ} \mathrm{C} ; \mathrm{UR}=42,1 \%$ \\
\hline 8. Glyphosate + 2,4-D $\left(960+335 \mathrm{~g} \mathrm{ha}^{-1}\right)$ & $12: 00 \mathrm{~h}$ & $\mathrm{~V}=5,3 \mathrm{~km} \mathrm{~h}^{-1} ;$ (poucas nuvens) \\
\hline 9. Glyphosate $\left(960 \mathrm{~g} \mathrm{ha}^{-1}\right)$ & $17.00 \mathrm{~h}$ & Temp $=24,1^{\circ} \mathrm{C} ; \mathrm{UR}=53,8 \%$ \\
\hline 10. Glyphosate $+2,4-\mathrm{D}\left(960+335 \mathrm{~g} \mathrm{ha}^{-1}\right)$ & $17: 00 \mathrm{~h}$ & $\mathrm{~V}=2,2 \mathrm{~km} \mathrm{~h}^{-1} ;$ (poucas nuvens) \\
\hline 11. Testemunha sem aplicação & - & -1 \\
\hline
\end{tabular}

Temp = Temperatura; UR = Umidade relativa do ar; V = Velocidade dos ventos. Todas as soluções herbicidas dos tratamentos foram aplicadas associadas ao marcado FDC-1 (1500 ppm).

As aplicações foram realizadas com pulverizador costal de pressão constante, utilizando quatro pontas de pulverização jato plano com indução de ar, modelo AVI 110.02, em velocidade de deslocamento de $3,6 \mathrm{~km} \mathrm{~h}^{-1}$, o que constituiu taxa de aplicação de $200 \mathrm{~L} \mathrm{ha}^{-1}$ com produção de gotas grossas, segundo norma ASAE S572 (informação do fabricante). No momento da aplicação as plantas de cipó-deveado apresentavam estádio de desenvolvimento de 3 a 5 folhas, assim como as condições climáticas foram monitoradas com anemômetro digital, em todos horários de aplicação estudados (Tabela 1).

As avaliações foram constituídas pela porcentagem de controle visual do cipó-deveado, utilizando-se 2 plantas por vaso, realizada aos 3, 7, 14, 21 e 28 DAA (dias após aplicação), conforme metodologia descrita pela (SBCPD, 1995), onde 0\% representou ausência de sintomas de injúrias nas plantas e $100 \%$ a morte das mesmas. Para avaliação da deposição da pulverização foram coletadas 2 plantas daninhas por vaso para recuperação do traçador FDC-1 Azul Brilhante (1500 ppm), o qual foi 
aplicado juntamente com as soluções herbicidas. Para recuperação do referido traçador da parte aérea das plantas foi realizada a lavagem das plantas com volume padronizado de $10 \mathrm{~mL}$ de água por planta, e posteriormente, utilizado procedimentos de espectrofotometria, com resultados em absorbância transformados em $\mu \mathrm{L}$ do traçador por planta, conforme metodologia descrita por Palladini et al. (2005), os quais mencionaram a viabilidade desse traçador por não alterar as características físicas da calda de pulverização.

Os dados obtidos foram submetidos à análise de variância e suas médias comparadas pelo teste de Scott-Knott a 5\% de probabilidade, e/ou por meio da comparação dos resultados por intervalo de confiança a $5 \%$ de probabilidade.

\section{Resultados e Discussão}

Para o controle da planta daninha cipóde-veado ( $P$. convolvulus) observou-se que de forma geral dos 3 aos 21 DAA todos os tratamentos com a associação de glyphosate + 2,4-D foram significativamente superiores ao glyphosate isolado, independentemente do horário da aplicação (Tabela 2). A partir dos 14 DAA, todos os tratamentos apresentaram níveis de controle da planta daninha considerados satisfatórios $(\geq 80 \%)$, conforme critérios da SBCPD (1995), com eficiência igual ou superior a $82,2 \%$.

Segundo Vidal et al. (2016), a associação de glyphosate com herbicidas mimetizadores de auxina, como por exemplo o 2,4-D, em geral resulta em efeito sinérgico, aumentando a eficiência de controle de plantas daninhas. Vários relatos são encontrados na literatura indicando esse efeito sinergístico para espécies eudicotiledôneas de difícil controle, como, buva, trapoeraba, corda-de-viola, poaiabranca e leiteiro (Carvalho et al., 2002; Oliveira Neto et al., 2010; Paula et al., 2011; Takano et al., 2013).

Tabela 2. Tratamentos constituídos pela aplicação de herbicidas em diferentes horários para o controle de cipó-de-veado aos 3, 7, 14, 21 e 28 DAA. Guarapuava (PR), 2015.

\begin{tabular}{|c|c|c|c|c|c|c|}
\hline \multirow{2}{*}{ Herbicidas (dose $\mathrm{g} \mathrm{ha}^{-1}$ ) } & \multirow{2}{*}{ Horários } & \multicolumn{5}{|c|}{ Controle (\%) } \\
\hline & & 3 DAA & $7 \mathrm{DAA}$ & $14 \mathrm{DAA}$ & $21 \mathrm{DAA}$ & $28 \mathrm{DAA}$ \\
\hline 1. Glyphosate (960) & & $12,6 \mathrm{c}$ & $16,2 \mathrm{~d}$ & $91,8 \mathrm{~b}$ & $98,6 \mathrm{a}$ & $99,6 \mathrm{a}$ \\
\hline 2. Glyphosate + 2,4-D $(960+335)$ & $22: 00 \mathrm{n}$ & $20,2 \mathrm{a}$ & $29,2 \mathrm{a}$ & 96,4 a & $99,0 \mathrm{a}$ & $99,8 \mathrm{a}$ \\
\hline 3. Glyphosate (960) & $2.00 \mathrm{~b}$ & $12,2 \mathrm{c}$ & $18,2 \mathrm{~d}$ & $88,2 \mathrm{c}$ & $98,2 \mathrm{a}$ & $99,0 \mathrm{a}$ \\
\hline 4. Glyphosate + 2,4-D $(960+335)$ & 5.0011 & $16,2 \mathrm{~b}$ & $23,2 \mathrm{~b}$ & $91,2 \mathrm{~b}$ & $99,6 \mathrm{a}$ & $100,0 \mathrm{a}$ \\
\hline 5. Glyphosate (960) & & $13,2 \mathrm{c}$ & $16,8 \mathrm{~d}$ & $88,8 \mathrm{c}$ & $99,2 \mathrm{a}$ & 99,8 a \\
\hline 6. Glyphosate + 2,4-D $(960+335)$ & 7:00 n & $20,8 \mathrm{a}$ & $24,6 \mathrm{~b}$ & $92,2 \mathrm{~b}$ & $100,0 \mathrm{a}$ & $100,0 \mathrm{a}$ \\
\hline 7. Glyphosate (960) & $12 \cdot 00 \mathrm{~h}$ & $10,0 \mathrm{c}$ & $13,4 \mathrm{e}$ & $82,2 \mathrm{~d}$ & $88,2 \mathrm{~d}$ & $93,2 \mathrm{~b}$ \\
\hline 8. Glyphosate + 2,4-D $(960+335)$ & 12.0011 & $14,8 \mathrm{~b}$ & $20,8 \mathrm{c}$ & $85,6 \mathrm{c}$ & $91,8 \mathrm{c}$ & $93,6 \mathrm{~b}$ \\
\hline 9. Glyphosate $\left(960 \mathrm{~g} \mathrm{ha}^{-1}\right)$ & & $11,8 \mathrm{c}$ & $14,2 \mathrm{e}$ & $90,2 \mathrm{~b}$ & $95,6 \mathrm{~b}$ & $98,8 \mathrm{a}$ \\
\hline 10. Glyphosate + 2,4-D $(960+335)$ & $1 /: 00 \mathrm{n}$ & $15,2 \mathrm{~b}$ & $23,6 \mathrm{~b}$ & $95,2 \mathrm{a}$ & 99,8 a & $100,0 \mathrm{a}$ \\
\hline 11. Testemunha sem aplicação & - & $0,0 \mathrm{~d}$ & $0,0 \mathrm{f}$ & $0,0 \mathrm{e}$ & $0,0 \mathrm{e}$ & $0,0 \mathrm{e}$ \\
\hline Fcal & & $31,50 *$ & $76,88^{*}$ & $344,27 *$ & $1788,73 *$ & $2490,70 *$ \\
\hline $\mathrm{CV}(\%)$ & & 16,59 & 10,85 & 4,04 & 1,77 & 1,49 \\
\hline
\end{tabular}

Médias seguidas das mesmas letras nas colunas não diferem entre si pelo teste de agrupamento de médias de Scott-Knott, (p $\leq 0,05)$.

Quanto aos horários das aplicações, os tratamentos com glyphosate isolado assim como glyphosate + 2,4-D aplicados as 12:00 h foram os que apresentaram os menores níveis de controle da planta daninha até os 28 DAA, apesar de todos os tratamentos estudados terem sido eficientes nesta última avaliação ( $\geq 93,3 \%)$
(Tabela 2). Estes resultados indicam que mesmo havendo controle eficiente em todos horários de aplicação aos 28 DAA, o período das 12:00 h foi o que provavelmente poderia resultar em menor eficiência se considerarmos outras a interação com outras variáveis não estudadas, tais como o tamanho de gotas aplicadas, tipo de formulações 
e tolerância da espécie a ser controlada aos herbicidas.

Os maiores níveis de matéria seca da parte aérea da planta daninha aos 28 DAA também confirmaram as informações de menor nível de controle para aplicação de glyphosate e glyphosate $+2,4-\mathrm{D}$ realizada as 12:00 $\mathrm{h}$ (Figura 1), uma vez que com exceção da testemunha sem aplicação, esse horário apresentou quantidade significativamente superior de matéria seca em relação aos demais horários.
Entretanto, é importante considerar que a aplicação realizada as 12:00 $\mathrm{h}$ foi a que ocorreu em condições atmosféricas as quais poderiam influenciar negativamente as boas práticas agronômicas na aplicação dos herbicidas, uma vez que apesar da temperatura $\left(26,3^{\circ} \mathrm{C}\right)$ e velocidade dos ventos $\left(5,3 \mathrm{~km} \mathrm{~h}^{-1}\right)$ estarem em níveis considerados adequados (Rodrigues e Almeida, 2011), no referido horário constituiu a menor umidade relativa do ar $(42,1 \%)$ (Tabela 1).

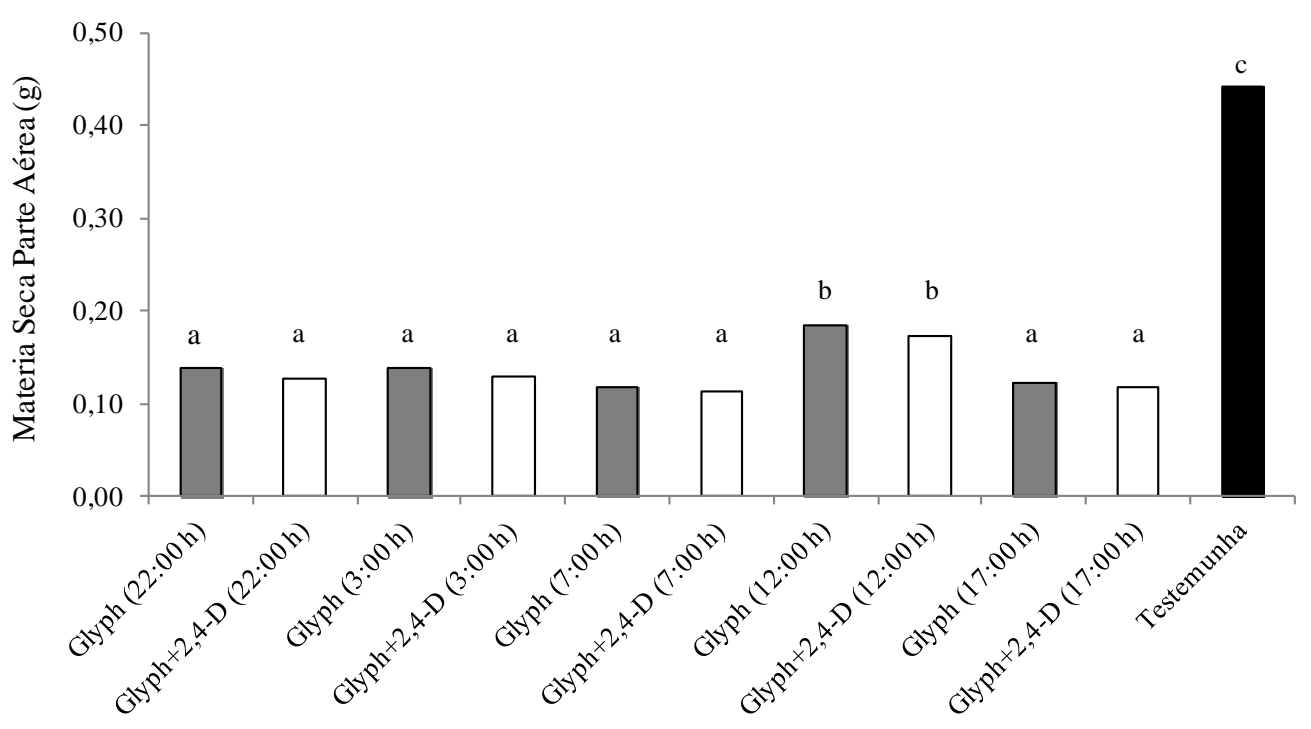

Figura 1. Matéria seca da parte aérea (g) de cipó-de-veado aos 28 DAA dos herbicidas glyphosate e glyphosate + 2,4-D, realizadas em diferentes horários. Guarapuava (PR), 2015.

Esses resultados indicam que condições climáticas inadequadas de umidade relativa do ar podem influenciar negativamente a qualidade da aplicação e, consequentemente, a eficiência no controle de plantas daninhas, corroborando com os resultados encontrados por Ferreira et al. (1998) e Ramires et al. (1999). Segundo esses autores os herbicidas lactofen, fomesafen e bentazon aplicados em horários com temperatura e umidade relativa do ar mais adequadas tiveram maior eficiência no controle de plantas daninhas. De forma contrária, Nunes et al. (2010) e Almeida et al. (2014) relataram que as condições atmosféricas encontradas nos diferentes horários de aplicação não limitaram a eficácia do glyphosate para o controle de
Urochloa plantaginea, Bidens sp. e Urochloa ruziziensis, respectivamente.

$\mathrm{Na}$ prática, o conhecimento de informações que relacionam a eficiência dos herbicidas com as condições climáticas no momento da aplicação são de extrema importância para o sucesso das aplicações, uma vez que além da dependência de condições ambientais favoráveis no período, existe também as interações específicas entre os herbicidas e as plantas daninhas. Nesse contexto, Azevedo (2015) relata que, de forma geral, os produtores agrícolas acreditam que o insucesso de uma aplicação está associado ao uso de tecnologia inadequada e não da observância das condições atmosféricas. 
Vale ressaltar que a necessidade de rendimento operacional de áreas extensas, associado a uma pequena janela com condições meteorológicas favoráveis, mais facilmente encontrada nos períodos de início e final da tarde, em muitos casos condiciona que as aplicações ocorram invariavelmente. Assim Nicolai e Christoffoleti (2014) sobre essa dinâmica operacional consideram que interação dos fatores meteorológicos é mais prejudicial do que quando considerados isolados, mas em ordem de importância na aplicação deve-se considerar inicialmente a velocidade dos ventos, depois a umidade relativa do ar e, por fim, a temperatura.

A associação em mistura em tanque de 2,4-D ao glyphosate, apesar de ter proporcionado melhor eficiência de controle das plantas de cipó-de-veado (Tabela 2), houve redução significativa na deposição do traçador da calda aplicada em relação ao glyphosate isolado, independentemente do horário de aplicação (Figura 2).

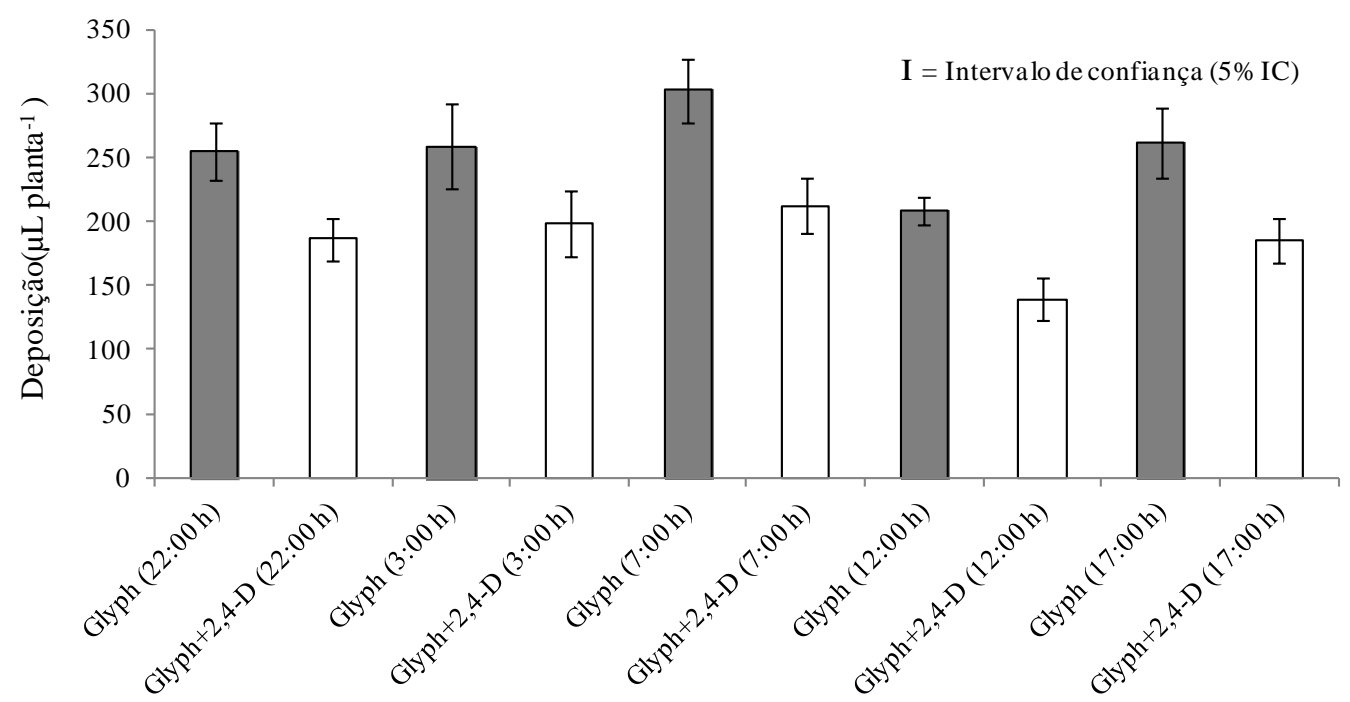

Figura 2. Deposição da calda de aplicação ( $\mu \mathrm{L}$ planta $\left.^{-1}\right)$ em cipó-de-veado dos herbicidas glyphosate e glyphosate +2,4-D, realizadas em diferentes horários. Guarapuava (PR), 2015.

No entanto, observou-se que a aplicação de glyphosate e glyphosate $+2,4-\mathrm{D}$ nas condições ambientais do horário das 12:00 h foi a que teve maior redução da deposição do traçador nas plantas de cipó-de-veado quando comparados ao demais horários. Portanto, a interação dessas variáveis pode ter contribuído para a ocorrência dos menores níveis de controle da planta daninha, sendo que entre uma das possíveis justificativas encontra-se a redução do espectro de gotas em condição de baixa umidade relativa do ar no horário das 12:00 h. Esses resultados corroboram com os descritos por Nascimento et al. (2012), os quais utilizando alvos artificiais constataram menor deposição da aspersão do glyphosate em horários quando as condições de temperatura e umidade relativa do ar são menos favoráveis às aplicações. Os autores também observaram que a aspersão de gotas finas proporciona menores deposições em comparação às gotas médias e grossas, e consideraram esse comportamento como esperado.

Ao utilizar a mesma metodologia de recuperação da solução traçadora, Marcon (2015) também encontraram reduções expressivas da deposição da pulverização em plantas de capim-amargoso (Digitaria insularis) e buva (Conyza bonariensis) utilizando associações dos herbicidas glyphosate + 
inibidor de ACCase com 2,4-D. Segundo o autor é difícil explicar a razão pela qual a quantidade de deposito da pulverização tenha sido influenciada negativamente pela presença de 2,4-D nas associações dos herbicidas. Entre as mais prováveis suposições de causa/efeito, estão a interferência do 2,4-D no espectro do diâmetro das gotas formadas pela aspersão e a possibilidade de absorção diferencial dos herbicidas. Entretanto, a razão para a tolerância diferencial da maioria das espécies de plantas daninhas não é ainda compreendida (Starke e Oliver, 1998).

Com base nos resultados obtidos, as informações de eficiência de controle e deposição da pulverização na planta daninha cipó-de-veado indicam que, com exceção dos tratamentos das $12: 00 \mathrm{~h}$, todos os demais horários de aplicação não foram influenciados pelas condições climáticas estudadas, possibilitando aplicações diurnas e noturnas dos herbicidas e uma análise mais criteriosa para quando as condições climáticas foram consideradas mais inadequadas. No entanto, novos experimentos deverão ser realizados para melhor compreensão dos dados obtidos, assim como para verificar se esse mesmo comportamento se apresenta para outras espécies de plantas daninhas.

\section{Conclusões}

Os herbicidas glyphosate e glyphosate + 2,4-D controlaram eficientemente o cipó-deveado (Polygonum convolvulus), independentemente do horário da aplicação, mesmo havendo menor deposição da pulverização no horário das 12:00 h.

Houve menor deposição na associação glyphosate + 2,4-D, em comparação ao glyphosate isolado, em todos os horários pesquisados.

\section{Agradecimentos}

Os autores agradecem ao $\mathrm{CNPq}$ e a Fundação Araucária pelo incentivo e concessão de bolsas de pós-graduação e iniciação científica PAIC-FA e PIBIC-EM.

\section{Referências}

Almeida, D.P.; Timossi, P.C.; Lima, S.F.; Silva, U.R.; Reis, E.F. Condições atmosféricas e volumes de aplicação na dessecação de Urochloa ruziziensis e vegetação espontânea. Revista Brasileira de Herbicidas, v.13, n.3, p.245-251, 2014.

Antuniassi, U.R. Qualidade em tecnologia de aplicação de defensivos. In: Congresso Brasileiro de Algodão, 5., 2005, Salvador. Anais... Campina Grande: Embrapa, 2005. p.6.

Azevedo, L.A.S. Tecnologia de aplicação para misturas em tanque. In: Azevedo, L.A.S. (Ed.). Misturas de tanque de produtos fitossanitários: teoria e prática. Rio de Janeiro: Editora IOMS Gráfica e Editora. 2015, v.1, cap.10, p.177-203.

Carvalho, F.T.; Pereira, F.A.R.; Peruchi, M.; Palazzo, R.R.B. Manejo químico das plantas daninhas Euphorbia heterophylla e Bidens pilosa em sistema de plantio direto da cultura de soja. Planta Daninha, v.20, n.1, p.145-150, 2002.

Cieslik, L.F.; Vidal, R.A.; Trezzi, M.M. Fatores ambientais que afetam a eficácia de herbicidas inibidores da ACCase: Revisão. Planta Daninha, v.31, n.2, p.483-489, 2013.

Cieslik, L.F.; Vidal, R.A.; Trezzi, M.M.; Machado, A.B.; Patel, F.; Xavier, E. et al. Condições ambientais da aplicação no início da manhã que favorecem a eficácia de fluazifop-pbutyl no controle de gramíneas. In: Congresso Brasileiro da Ciência das Plantas Daninhas, 28., 2012, Campo Grande. Anais... Campo Grande: SBCPD, 2012. p.137-141. (CD-ROM)

Cunha, J.P.A.R.; Pereira, J.N.P.; Barbosa, L.A.; Silva, C.R. Pesticide application windows in the region of Uberlândia-MG. Bioscience Journal, v.32, n.2, p.403-411, 2016. 
Ferreira, M.C.; Machado Neto, J.G.; Matuo, T. Redução da dose e volume de calda nas aplicações noturnas de herbicidas em pósemergência na cultura da soja. Planta Daninha, v.6, n.1, p.25-36, 1998.

Kissmann K.G.; Groth D. Plantas infestantes e Nocivas: Tomo III. 2. ed., São Paulo: Basf Brasileira S.A., p.295-298, 2000.

Marcon, J.E.L. Misturas em tanque de herbicidas como estratégias de manejo de plantas daninhas resistentes ao glyphosate na cultura da soja $\mathbf{R R}^{\circledR}$. 56f. Dissertação (Mestrado em Agronomia) - Unversidade Estadual de Maringá, Umuarama, 2015.

Matthews, G.A. Pesticide Application Methods. 3.ed. London: Willey-Blackwell, 2000. 448p.

Moreira, H.J.C.; Bragança, H.B.N. Manual de identificação de plantas infestantes: Hortifrúti. São Paulo: FMC Agricultural Products, p.788-789, 2011.

Nascimento, A.B.; Oliveira, G.M.; Balan, M.G.; Higashibara, L.R.; Abi Saab, O.J.G. Deposição de glifosato e utilização de adjuvante para diferentes pontas de pulverização e horário de aplicação. Revista Brasileira de Tecnologia Aplicada nas Ciências Agrárias, v.5, n.2, p.105-116, 2012.

Nicolai, M.; Christoffoleti, P.J. Tecnologia de aplicação de herbicidas. In: Monquero, P.A. Aspectos da biologia e manejo das plantas daninhas. São Carlos: RiMa. 2014, p.307-350.

Nunes, A.L.; Merotto Júnior, A.; Vidal, R.A.; Goulart, I.C.G.; Kupas, V. Variáveis meteorológicas para determinação do efeito do momento de aplicação de herbicida na cultura da soja. In: Congresso Brasileiro da Ciência das Plantas Daninhas, 27., 2010, Ribeirão Preto. Anais... Ribeirão Preto: SBCPD, 2010. p.507510. (CD-ROM)

Oliveira Neto, A.M.; Guerra, N.; Dan, H.A.; Braz, G.B.P.; Jumes, T.M.C.; Santos, G. et al. Manejo de Conyza bonariensis com glyphosate
+ 2,4-D e amônio-glufosinate em função do estádio de desenvolvimento. Revista Brasileira de Herbicidas, v.9, n.3, p.73-80, 2010.

Palladini, L.A.; Raetano, C.G.; Velini, E.D. Choice of tracers for the evaluation of spray deposits. Scientia Agricola, v.62, n.5, p.440445, 2005.

Paula, J.M.; Vargas, L.; Agostinetto, D.; Nohatto, M.A. Manejo de Conyza bonariensis resistente ao herbicida glyphosate. Planta Daninha, v.29, n.1, p.217-227, 2011.

Ramires, A.C.; Constantin, J.; Marchiori Júnior, O.; Maciel, C.D.G.; Oliveira Júnior, R.S.; Apoloni, D.K.M. Influência dos diferentes horários de aplicação em pós-emergência dos herbicidas chlorimuron-ethyl, fomesafen e bentazon no controle de Commelina benghalensis L. Acta Scientiarum, v.21, n.3, p.467-472, 1999.

Rodrigues, B.N.; Almeida, F.S. Guia de herbicidas. Londrina: IAPAR, 2011. 697p.

SOCIEDADE BRASILEIRA DA CIÊNCIA DAS PLANTAS DANINHAS - SBCPD. Procedimentos para instalação, avaliação e análise de experimentos com herbicidas. $1^{\mathrm{a}}$ ed. Londrina: SBCPD, 1995. 42p.

Starke, R.J.; Oliver, L.R. Interaction of glyphosate with chlorimuron, fomesafen, imazethapyr and sulfentrazone. Weed Science, v.46, n.6, p.652-660, 1998.

Takano, H.K.; Oliveira Júnior, R.S.; Constantin, J.; Biffe, D.F.; Franchini, L.H.M.; Braz, G.B.P. et al. Efeito da adição do 2,4-D ao glyphosate para o controle de espécies de plantas daninhas de difícil controle. Revista Brasileira de Herbicidas, v.12, n.1, p.1-13, 2013.

Vidal, R.A.; Queiroz, A.R.S.; Trezzi, M.M.; Kruse, N.D. Association of glyphosate with other agrochemicals: the knowledge synthesis. Revista Brasileira de Herbicidas, v.15, n.1, p.39-47, 2016. 